\title{
Islet cell dedifferentiation is a pathologic mechanism of long-standing progression of type 2 diabetes
}

\author{
Kikuko Amo-Shiinoki, ${ }^{1,2}$ Katsuya Tanabe, ${ }^{1}$ Yoshinobu Hoshii, ${ }^{3}$ Hiroto Matsui, ${ }^{4}$ Risa Harano, ${ }^{1}$ \\ Tatsuya Fukuda, ${ }^{5}$ Takato Takeuchi, ${ }^{5}$ Ryotaro Bouchi, ${ }^{5,6}$ Tokiyo Takagi, ${ }^{1}$ Masayuki Hatanaka, \\ Komei Takeda, ${ }^{1}$ Shigeru Okuya, ${ }^{7}$ Wataru Nishimura, ${ }^{8}$ Atsushi Kudo, ${ }^{9}$ Shinji Tanaka, ${ }^{10}$ \\ Minoru Tanabe, ${ }^{9}$ Takumi Akashi, ${ }^{11}$ Tetsuya Yamada, ${ }^{5}$ Yoshihiro Ogawa, ${ }^{5,12,13,14}$ Eiji Ikeda, ${ }^{15}$ \\ Hiroaki Nagano, ${ }^{4}$ and Yukio Tanizawa'
}

'Division of Endocrinology, Metabolism, Hematological Sciences and Therapeutics, Department of Medicine, Yamaguchi University Graduate School of Medicine, Ube, Yamaguchi, Japan. ${ }^{2}$ Department of Diabetes Research, Yamaguchi University School of Medicine, Ube, Yamaguchi, Japan. ${ }^{3}$ Department of Diagnostic Pathology, Yamaguchi University Hospital, Ube, Yamaguchi, Japan. ${ }^{4}$ Department of Gastroenterological, Breast and Endocrine Surgery, Yamaguchi University Graduate School of Medicine, Ube, Yamaguchi, Japan. ${ }^{5}$ Department of Molecular Endocrinology and Metabolism, Graduate School of Medical and Dental Sciences, Tokyo Medical and Dental University, Tokyo, Japan. ${ }^{6}$ Department of Diabetes, Endocrinology and Metabolism, National Center for Global Health and Medicine, Tokyo, Japan. ${ }^{7}$ Health Administration Center, Yamaguchi University Organization for University Education, Yamaguchi, Japan. ${ }^{8}$ Department of Molecular Biology, International University of Health and Welfare School of Medicine, Chiba, Japan. ${ }^{9}$ Department of Hepatobiliary and Pancreatic Surgery, ${ }^{10}$ Department of Molecular Oncology, "Department of Diagnostic Pathology, and ${ }^{12}$ Department of Molecular and Cellular Metabolism, Graduate School of Medical and Dental Sciences, Tokyo Medical and Dental University, Tokyo, Japan. ${ }^{13}$ Department of Medicine and Bioregulatory Science, Graduate School of Medical Sciences, Kyushu University, Fukuoka, Japan. ${ }^{14}$ AMED-CREST, Tokyo, Japan. ${ }^{15}$ Department of Pathology, Yamaguchi University Graduate School of Medicine, Ube, Yamaguchi, Japan.

Authorship note: KAS and K Tanabe contributed equally to this study.

Conflict of interest: The authors have declared that no conflict of interest exists.

Copyright: ( 2021 , Amo-Shiinoki et al. This is an open access article published under the terms of the Creative Commons Attribution 4.0 International License.

Submitted: September 2, 2020 Accepted: November 18, 2020 Published: January 11, 2021

Reference information: JCI Insight. 2021:6(1):e143791.

https://doi.org/10.1172/jici. insight.143791.
Dedifferentiation has been implicated in $\beta$ cell dysfunction and loss in rodent diabetes. However, the pathophysiological significance in humans remains unclear. To elucidate this, we analyzed surgically resected pancreatic tissues of 26 Japanese subjects with diabetes and 11 nondiabetic subjects, who had been overweight during adulthood but had no family history of diabetes. The diabetic subjects were subclassified into 3 disease stage categories, early, advanced, and intermediate. Despite no numerical changes in endocrine cells immunoreactive for chromogranin A (ChgA), diabetic islets showed profound $\beta$ cell loss, with an increase in $\alpha$ cells without an increase in insulin and glucagon double-positive cells. The proportion of dedifferentiated cells that retain ChgA immunoreactivity without 4 major islet hormones was strikingly increased in diabetic islets and rose substantially during disease progression. The increased dedifferentiated cell ratio was inversely correlated with declining C-peptide index. Moreover, a subset of islet cells converted into exocrine-like cells during disease progression. These results indicate that islet remodeling with dedifferentiation is the underlying cause of $\beta$ cell failure during the course of diabetes progression in humans.

\section{Introduction}

Diabetes mellitus (DM) is a metabolic disorder characterized by loss and dysfunction of pancreatic $\beta$ cells along with insulin resistance, resulting in multiple long-term complications $(1,2)$. Despite various oral therapies to lower glucose, disease progression is associated with a profound decline in $\beta$ cell function leading to consequent insulin replacement $(3,4)$. Although it is accepted that increased apoptosis plays a role in declining $\beta$ cell function and mass over time (5-8), an increase in apoptosis alone is insufficient to explain the $\beta$ cell deficit in type 2 diabetes $(9,10)$. Additionally, in human islet-to-mouse studies, neither chronic hyperglycemia nor peripheral insulin resistance was sufficient to cause apoptosis in human islets (11). 
Definitive lineage-tracing experiments in rodent diabetes have recently identified $\beta$ cell dedifferentiation with reversion to a progenitor-like state $(12,13)$. Importantly, analyses of human cadaveric pancreatic tissues have provided corroboration of the results of animal studies $(14,15)$. A dedifferentiated $\beta$ cell is defined as one that no longer contains pancreatic hormones, although it retains endocrine features such as chromogranin $\mathrm{A}$ and/or synaptophysin immunoreactivity $(12,14)$. The fact that islet hormone-negative endocrine cells were significantly increased in postmortem diabetic specimens suggests $\beta$ cell dedifferentiation to be involved in insufficient insulin secretion (14). However, the information from cadaveric sample analyses does not account for either the extent or the mechanism by which $\beta$ cell dedifferentiation contributes to the $\beta$ cell deficit and its progression during the entire disease course of diabetes. Elucidating this issue is a key challenge owing to the difficulty in obtaining long-term access to human pancreatic samples of appropriate quality for analyses, in conjunction with the necessary medical history and metabolic profiles. Therefore, we took advantage of the opportunity to examine pancreatic samples from Japanese patients with and without diabetes undergoing partial pancreatectomy, for resection of pancreaticobiliary neoplasms, to examine the hypothesis that islet remodeling with dedifferentiation is a pathologic mechanism underlying long-standing disease progression. To this end, we subclassified the subjects with diabetes into early- and advanced-stage disease and examined islet morphology and dedifferentiation, as well as correlations with clinical parameters. Our work reveals the involvement of dedifferentiation in $\beta$ cell dysfunction and loss during the course of diabetes progression.

\section{Results}

Clinical data and morphometric measurements. Clinical characteristics of the 11 control patients (non-DM) and 26 diabetic patients subclassified into 3 categories are summarized in Table 1, and clinical feature of each individual subject is shown in Supplemental Table 1 (supplemental material available online with this article; https://doi.org/10.1172/jci.insight.143791DS1). All study groups included patients with pancreatic cancer, and there were several with cholangiocarcinoma and duodenal papilla cancer among the non-DM and early- and advanced-DM patients. The non-DM and intermediate-DM patients were younger than those in the other 2 groups, although the mean ages did not differ significantly, whereas mean ages were similar in the early- and advanced-DM patients. Mean maximum BMI differed minimally among groups. However, BMI decreased with disease progression and was significantly lower in the advanced-DM than in the non-DM subjects $(P=0.031)$. Fasting plasma glucose and glycated hemoglobin $\mathrm{A} 1 \mathrm{c}(\mathrm{HbA} 1 \mathrm{c})$ levels in the non-DM patients showed normal glucose tolerance. Durations of diabetes in the early-, advanced-, and intermediate-DM subjects were $4.2 \pm 0.9,17.5 \pm 5.6$, and $8.0 \pm 1.4$ years, respectively. The preoperative HbA1c levels were $6.7 \% \pm 0.4 \%$ (50 $\pm 5.5 \mathrm{mmol} / \mathrm{mol}), 7.3 \% \pm 0.7 \%(57 \pm 7.9 \mathrm{mmol} / \mathrm{mol})$, and $7.3 \% \pm$ $0.9 \%$ (56 $\pm 10.1 \mathrm{mmol} / \mathrm{mol})$, respectively. There were no statistically significant differences in HbA1c levels among the diabetic groups. As diabetes progressed, fasting plasma glucose levels rose and C-peptide immunoreactivity (CPR) levels decreased, resulting in a profound C-peptide index (CPI) reduction in patients with advanced DM $(P=0.0005$ vs. non-DM).

Subjects with diabetes had a broad range of fractional $\beta$ cell, $\alpha$ cell, and islet areas and $\alpha$ cell/ $\beta$ cell ratios as compared with non-DM subjects, but the differences among all groups did not reach statistical significance (Supplemental Figure 1, A-D). Although not statistically significant, islet areas in subjects with diabetes tended to be increased in the body to tail as compared with the head of the pancreas ( $P=$ 0.057 ) (Supplemental Figure 1E). There were significant correlations among $\beta$ cell, $\alpha$ cell, and islet areas $\left(r=0.818, P=3.431 \times 10^{-9} ; r=0.863, P=5.375 \times 10^{-11}\right.$, respectively) (Supplemental Figure 1, F and G). There was also a significant correlation between the $\beta$ cell and $\alpha$ cell areas $\left(r=0.717, P=1.802 \times 10^{-6}\right)$ (Supplemental Figure $1 \mathrm{H}$ ). In addition, there was a weak but statistically significant correlation between the $\alpha$ cell/ $\beta$ cell ratio and islet area $(r=0.378, P=0.028)$ (Supplemental Figure 1I).

Diabetic islets have preserved endocrine cells but show altered $\beta$ cell and $\alpha$ cell fractions. The large deviations in islet morphometrics in subjects with diabetes may reflect diverse capabilities for islet compensation in response to metabolic demands. To gain pathologic insight into failing islets, we examined the morphology of individual islets. Representative images demonstrate changes in the appearance of size-matched islets stained with chromogranin A (ChgA) and insulin or Gcg, with disease progression (Figure 1, A and B). ChgA-positive cells per islet numbers were similar in all groups (Figure 1C). Because of the small number of subjects, we did not include the intermediate-DM group in further comparisons. We detected a $34 \%$ decrease (from $76 \%$ to $50 \%$ ) and a $44 \%$ decrease (from $76 \%$ to $42 \%$ ) 
Table 1. Clinical characteristics of study subjects

\begin{tabular}{|c|c|c|c|c|}
\hline & Non-DM & Early-DM & Advanced-DM & Intermediate-DM \\
\hline$N$ (male/female) & $11(9 / 2)$ & $12(11 / 1)$ & $11(10 / 1)$ & $3(2 / 1)$ \\
\hline Operative procedure, $\mathrm{PD} / \mathrm{DP}$ & $6 / 5$ & $5 / 7$ & $7 / 4$ & $1 / 2$ \\
\hline Pancreatic cancer & 2 & 5 & 4 & 1 \\
\hline Cholangiocarcinoma & 2 & 3 & 2 & 0 \\
\hline Gallbladder cancer & 0 & 0 & 1 & 0 \\
\hline Neuroendocrine tumor & 3 & 1 & 0 & 1 \\
\hline IPMN & 2 & 1 & 3 & 1 \\
\hline Age, years & $61.0 \pm 12.1$ & $69.3 \pm 7.0$ & $67.8 \pm 10.7$ & $60.3 \pm 16.6$ \\
\hline Diabetes duration, years & $\mathrm{N} / \mathrm{A}$ & $4.2 \pm 0.9^{c}$ & $17.5 \pm 5.6$ & $8.0 \pm 1.4$ \\
\hline Maximum BMI, kg/m² & $27.9 \pm 2.7$ & $26.5 \pm 1.2$ & $27.3 \pm 2.1$ & $28.9 \pm 2.7$ \\
\hline $\mathrm{F}-\mathrm{CPR}, \mathrm{nmol} / \mathrm{L}$ & $0.72 \pm 0.29$ & $0.55 \pm 0.10$ & $0.43 \pm 0.24$ & $0.44 \pm 0.08$ \\
\hline $\mathrm{CPI}, \mathrm{nmol} / \mathrm{mmol}$ & $0.13 \pm 0.05$ & $0.08 \pm 0.02$ & $0.05 \pm 0.03^{B}$ & $0.07 \pm 0.01^{A}$ \\
\hline
\end{tabular}

Values are expressed as means $\pm \mathrm{SD}$. One-way ANOVA followed by Bonferroni's post hoc test. ${ }^{A} P<0.05$ vs. non-DM. ${ }^{B} P<0.01$ vs. non-DM. ${ }^{C} P<$ 0.05 vs. advanced-DM. ${ }^{\mathrm{D} P}<0.01$ vs. advanced-DM. CPI, C-peptide index; DP, distal pancreatectomy; FPG, fasting plasma glucose; F-CPR, fasting c-peptide immunoreactivity; IPMN, intraductal papillary mucinous neoplasia; PD, pancreatoduodenectomy.

in $\beta$ cells/islet ratio in the early-DM and advanced-DM groups, respectively, as compared with nonDM subjects $(P<0.0001, P<0.0001$ ) (Figure 1D). The $\alpha$ cells/islet ratio increased by $58 \%$ (from $33 \%$ to $52 \%$ ) and $73 \%$ (from $33 \%$ to $57 \%$ ) in the early-DM and advanced-DM groups, respectively ( $P$ $=0.007, P<0.0001$ ), leading to a higher $\alpha$ cell $/ \beta$ cell ratio per islet (Figure $1, \mathrm{E}$ and $\mathrm{F}$ ). Contrary to a previous report (16), the ratio of insulin/Gcg double-positive cells, when normalized by the number of ChgA-positive cells, was significantly decreased in diabetic groups as compared with non-DM subjects (Figure $1 G$ ). In addition, the mean ratio of advanced-DM was further decreased, by $55 \%$, as compared with that of early-DM, suggesting that cells coexpressing immunoreactive insulin and glucagon were hardly detectable in islets of diabetic subjects. Next, we sought to determine whether there are any functional correlations with alterations in islet morphology. We found a significant correlation between a decrease in $\beta$ cell/islet ratio and decreased CPI $(r=0.520, P=0.027)$ (Figure $1 \mathrm{H})$. In contrast, the $\alpha$ cell/islet ratio and the $\alpha$ cell/ $\beta$ cell ratio per islet both showed inverse correlations with CPI ( $r=-0.631, P=0.005 ; r=-0.563, P=0.015$, respectively) (Figure $1, \mathrm{I}$ and $\mathrm{J}$ ).

Islet cell dedifferentiation is involved in diabetes progression. We next sought to establish a link between dedifferentiation and loss and/or dysfunction of $\beta$ cells during the course of the disease. We arbitrarily defined a dedifferentiated cell as a ChgA-positive cell that failed to react with the antibodies against the aforementioned $4 \mathrm{H}$ (Figure 2A). Ghrelin, another pancreatic hormone, was separately tested. The percentages of cells immunoreactive for ghrelin in ChgA-positive cells was less than $1 \%$ without a significant change among study groups (data not shown). We next determined the numbers of dedifferentiated cells per islet in all study subjects. As shown in Figure 1C, there was no loss of cells corresponding to the general endocrine features in type 2 diabetes. The percentages of cells positive for $\mathrm{ChgA}$ and negative for $4 \mathrm{H}$ rose progressively, being lowest in the non$\mathrm{DM}$, then rising from early-DM, through intermediate-DM, and finally being highest in the advanced-DM specimens ( $4 \% \pm 2 \%$ vs. $16 \% \pm 5 \%, 21 \% \pm 7 \%, 25 \% \pm 7 \%$, respectively) $(P<0.001)$ (Figure $2 \mathrm{~B})$. Percentages of dedifferentiated cells (dedifferentiation score) in intermediate-DM and advanced-DM were higher by $37 \%$ and $59 \%$ versus the early-DM group, respectively $(P=0.618, P=0.0016$, respectively). There was no significant difference in dedifferentiation scores between the head and body to the tail of pancreas (Supplemental Figure 2A). We also assessed insulin-positive cells immunoreactive for nuclear V-maf musculoaponeurotic fibrosarcoma oncogene homolog A (MAFA) and NK homeobox, family 6, member A (NKX6.1), in a subset 

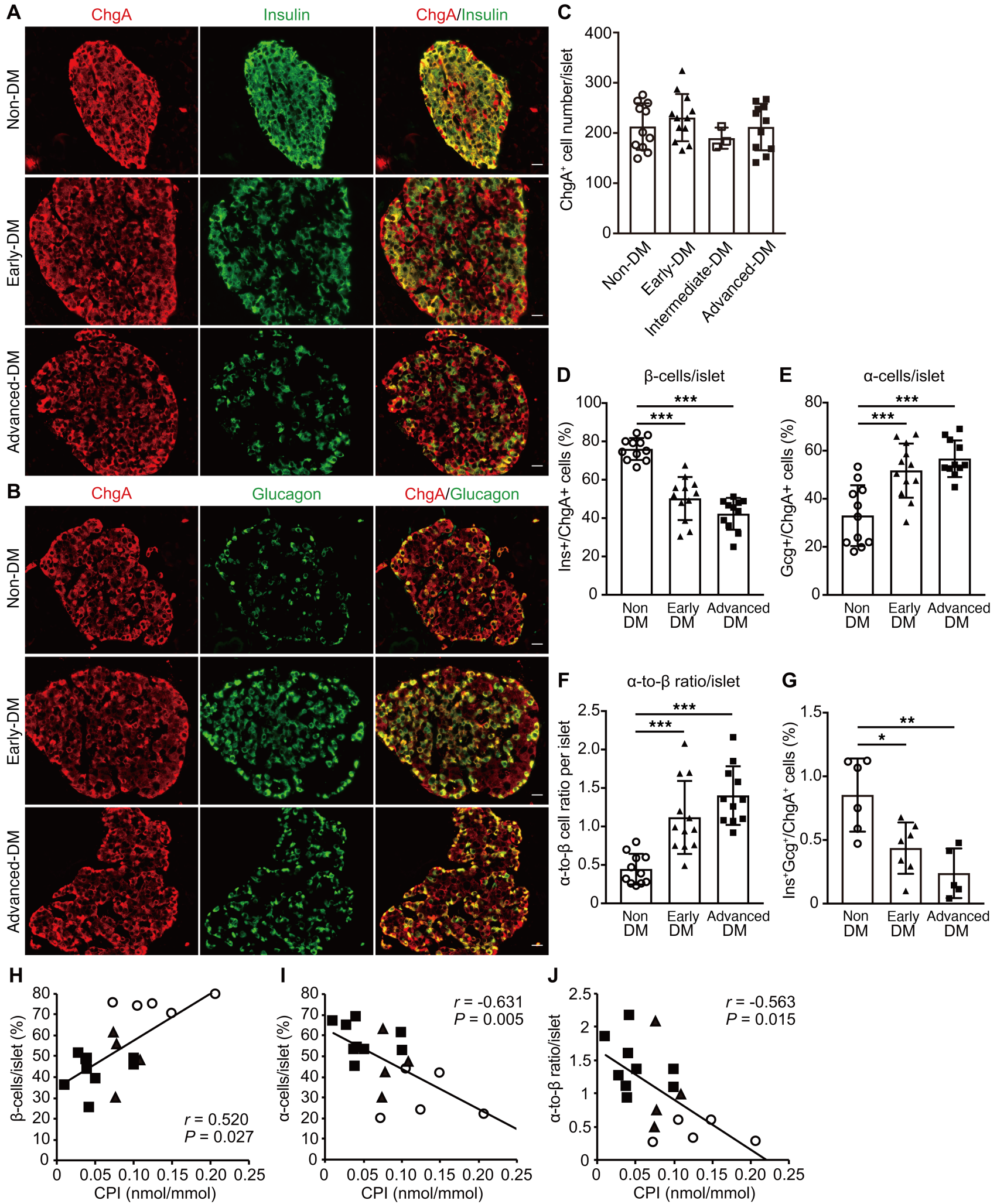

Figure 1. Morphologic changes in individual islets and correlation with $\boldsymbol{\beta}$ cell function. Representative images of pancreatic islets immunostained with chromogranin A (ChgA) (shown in red) and (A) insulin (shown in green) and (B) glucagon (shown in green) in each group. Scale bar: $20 \mu \mathrm{m}$. Quantitative analyses of (C) ChgA-positive cells per islet, (D) $\beta$ cells per islet, (E) $\alpha$ cells per islet, and (F) the $\alpha$ cell/ $\beta$ cell ratio per islet. Data are means $\pm \mathrm{SD}\left(n=11\right.$ for non-DM, $n=12$ for early-DM, $n=11$ for advanced-DM, and $n=3$ for intermediate-DM). ${ }^{* *} P<0.001$ by 1 -way ANOVA followed by Bonferroni's post hoc test. (G) Quantitative analyses of insulin and glucagon double-positive cells in the pancreatic sections of specimens from the indicated subjects in each group. Data are means \pm SD ( $n=6$ for non-DM, $n=7$ for early-DM, and $n=5$ for advanced-DM). ${ }^{*} P<0.05,{ }^{*} P<0.01$ by 1-way ANOVA followed by Bonferroni's post hoc test. We performed single regression analysis (Spearman's correlation coefficient) to assess 
relationships between the C-peptide index obtained from 18 subjects ( $n=5$ for non-DM, $n=4$ for early-DM, and $n=9$ for advanced-DM) and (H) $\beta$ cells/islet, (I) $\alpha$ cells/islet, and (J) $\alpha$ cell/ $\beta$ cell ratio per islet. Open circles, non-DM control subjects. Closed triangles, early-DM subjects. Closed squares, advanced-DM subjects. CPI, C-peptide index.

of subjects and found similar declines in early- and advanced-DM (Supplemental Figure 2, B, C, E, and F). When normalized by the number of ChgA-positive cells, although no significant differences were seen among the diabetic groups, there was a tendency for nuclear MAFA and NKX6.1, immunoreactivity to decrease with disease progression (Supplemental Figure 2, D and G). Notably, immunoreactivities for nuclear MAFA and NKX6.1 were weaker in advanced- than in early-DM specimens, demonstrating a loss of a mature cellular identity in parallel with dedifferentiation. In all study groups, dedifferentiation scores correlated strongly with a decreased $\beta$ cell/islet ratio $\left(r=-0.801, P=1.297 \times 10^{-8}\right)$ and an increased $\alpha$ cell/islet ratio $(r=0.642, P=$ $4.333 \times 10^{-5}$ ) (Figure 2, C and D). The morphologic correlations were further confirmed by immunostaining with insulin and other pancreatic hormones separately (Supplemental Figure 3A). Functional relevance was next examined. Dedifferentiation scores inversely correlated with CPI in all study groups $(r=-0.569, P=$ 0.007) (Figure 2E), and a similar inverse correlation was shown in the diabetic subjects except those with advanced-DM ( $r=-0.7928, P=0.0397)$ (Supplemental Table 2). As to clinical relevance, whereas no significant correlation between dedifferentiation scores and disease duration was identified for each diabetic group (Supplemental Table 2), dedifferentiation scores correlated significantly with disease duration when tested in whole subjects with diabetes (Figure 2F). In 13 subjects, there was no difference in dedifferentiation score between treating with insulin and with sulfonylurea (Figure $2 \mathrm{G}$ ). In addition, there was a significant correlation between BMI prior to operation and dedifferentiation score in nondiabetic control subjects $(r=-0.676, P$ $=0.026$ ), whereas it was not seen in those with diabetes (Supplemental Figure 3, B-D).

Correlations of dedifferentiation with islet morphology and age in early-DM subjects. $\beta$ cell dysfunction with a background of insulin resistance causes hyperglycemia that would in turn promote $\beta$ cell dedifferentiation (12-14, 17-19). However, other factors that might be involved are not fully understood. To explore this issue, we focused on 12 early-DM subjects, who would presumably have fewer long-standing effects of hyperglycemia than patients with longer disease durations. As shown in Figure 3A, we found that an increased islet area correlated significantly with $\alpha$ cell/islet ratio rather than $\beta$ cell/islet ratio in early-DM, resulting in an increase in the $\alpha$ cell/ $\beta$ cell ratio per islet. A significant correlation between dedifferentiation score and $\alpha$ cell/islet ratio was demonstrated $(r=0.644, P=0.022)$. However, no increase in the frequency of interconversion between $\beta$ cells and $\alpha$ cells, partly represented by insulin/Gcg double-positive cells, was identified in diabetic patients (Figure 1G), raising the possibility of an indirect correlation. Therefore, this series of observations implied that islet area expansion, assumed to occur before progression of hyperglycemia, would be accompanied by islet remodeling owing to islet cell plasticity. This would result in $\alpha$ cells being increased and thereby correlating indirectly with dedifferentiation. We next tested correlations with age (Figure 3B). All tests on control subjects showed there were no correlations between islet morphometries and age. Although there was no significant correlation between $\beta$ cells/islet and age, we found $\alpha$ cell/ islet ratio and $\alpha$ cell/ $\beta$ cell ratio per islet to correlate inversely with age. Moreover, dedifferentiation scores were decreased in older subjects and appeared to be inversely associated with age, although the relationship did not reach statistical significance $(r=-0.522, P=0.084)$.

Advanced stage of diabetes and altered islet cell fate. We then sought to examine features of dedifferentiated cells in the advanced disease stage. Tissues of islet area-matched male subjects without pancreatic cancer, whose details are summarized in Supplemental Table 3, were costained with ChgA and a nonendocrine marker, i.e., amylase, and the results were then compared among all groups. In 3 sets classified with different islet areas (Figure 4, A-C, D-F, and G-I, respectively), all advanced-DM subjects exhibited a subset of islet ChgA-positive cells immunoreactive for amylase (Figure 4, C, F, and I). Z-stack analysis demonstrated that amylase and ChgA were expressed in virtually the same cells (Figure 4J). An early-DM subject, who had the highest dedifferentiation score in this group, had similar cellular findings (Figure $4 \mathrm{H}$ ). Notably, such cells showed apparently decreased immunoreactivities for ChgA as compared with neighboring amylase-negative cells in the same islet, suggesting that a subset of dedifferentiated cells loses endocrine features and takes an exocrine fate. Although a previous study demonstrated a mesenchymal marker, vimentin-expressing cells emerging in diabetic donor islets (20), no islet cells immunoreactive for vimentin were detectable in our cases (data not shown). In contrast to early-DM (Figure 3A), the $\alpha$ cells/islet ratio in advanced-DM tended to show an inverse association with dedifferentiation score (Supplemental Figure 4A). 
A

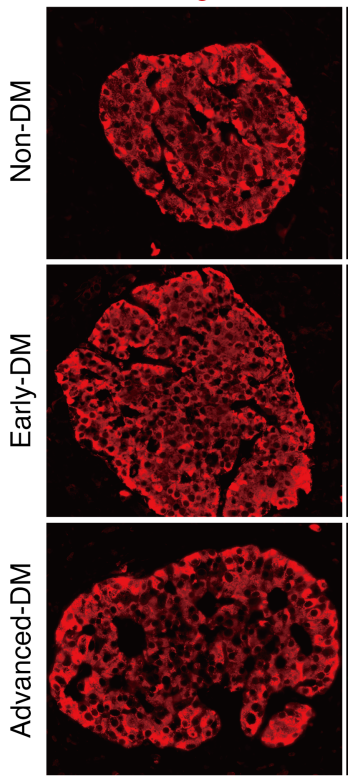

C

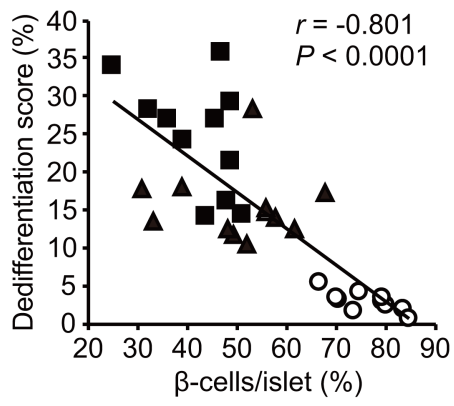

E

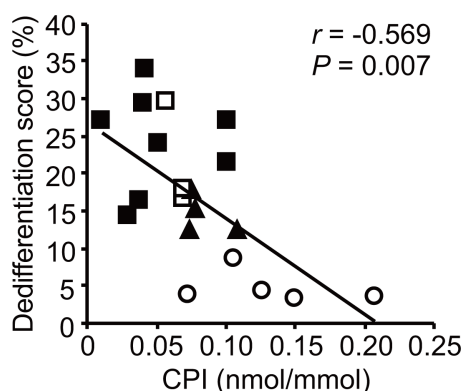

$4 \mathrm{H}$
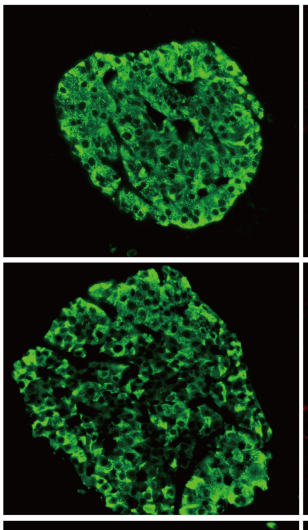
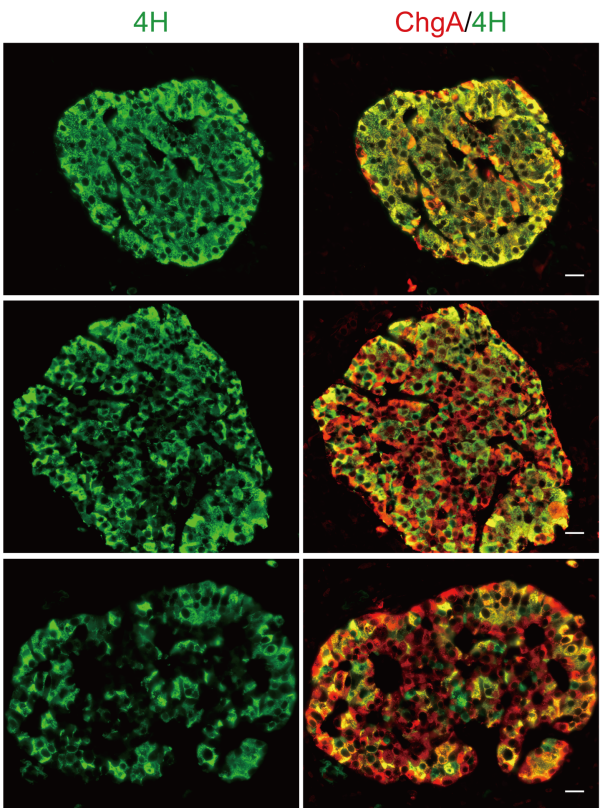

B Dedifferentiation score

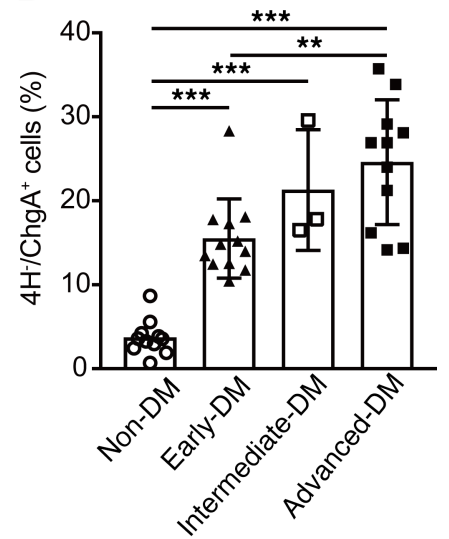

D

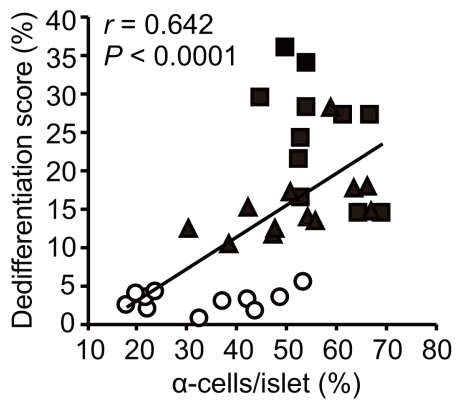

F

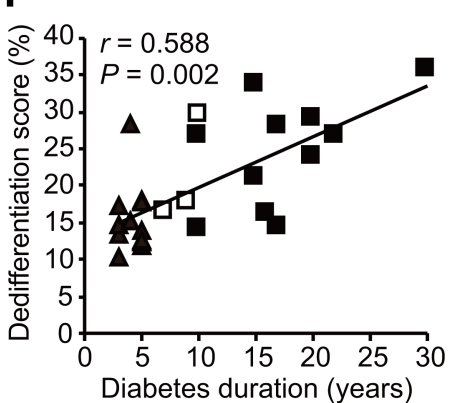

G

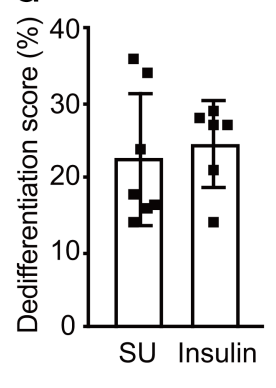

Figure 2. Evaluation of dedifferentiation and correlations with islet morphology and clinical parameters. (A) Representative images of pancreatic islets immunostained with ChgA (shown in red) and endocrine cocktail (insulin, glucagon, somatostatin, and pancreatic polypeptide [4H], shown in green). Scale bar: $20 \mu \mathrm{m}$. (B) Quantitative analysis of dedifferentiated cells (dedifferentiation score), calculated as ChgA $4 \mathrm{H}^{-} / \mathrm{ChgA}^{+}$cells per islet. Data are means $\pm \mathrm{SD}$. ${ }^{* *} P<0.01,{ }^{* * *} P<0.001$ by 1-way ANOVA followed by Bonferroni's post hoc test ( $n=11$ for non-DM, $n=12$ for early-DM, $n=3$ for intermediate-DM, and $n=11$ for advanced-DM). Single regression analysis (Spearman's correlation coefficient) of correlations between dedifferentiation score and (C) $\beta$ cells/ islet, (D) $\alpha$ cells/islet, and (E) the C-peptide index obtained from 21 subjects ( $n=5$ for non-DM, $n=4$ for early-DM, $n=3$ for intermediate-DM, and $n=9$ for advanced-DM), and (F) diabetes duration. (C) Comparison of dedifferentiation score between the subjects receiving insulin treatment $(n=6)$ and those treated with sulfonylurea but not insulin $(n=7)$. Open circles, control subjects. Closed triangles, early-DM subjects. Open squares, intermediate-DM subjects. Closed squares, advanced-DM subjects. CPI, C-peptide index. SU, sulfonylurea.

Immunohistochemical analysis using a specific anti-glucagon-like peptide-1 (GLP-1) antibody, which is highly selective for processed amidated GLP-1 directed to the COOH-terminal (21), demonstrated that glucagon colocalizes with GLP-1 and that glucagon immunoreactivities relative to GLP-1 decreased with disease progression (Supplemental Figure 4B). This immunohistochemical evidence suggested that both $\beta$ cell and $\alpha$ cell identity may be impaired over the course of disease progression. 
A

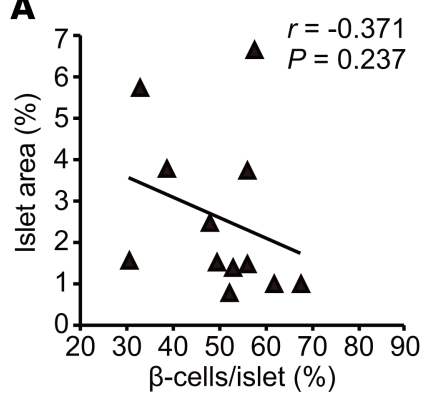

B
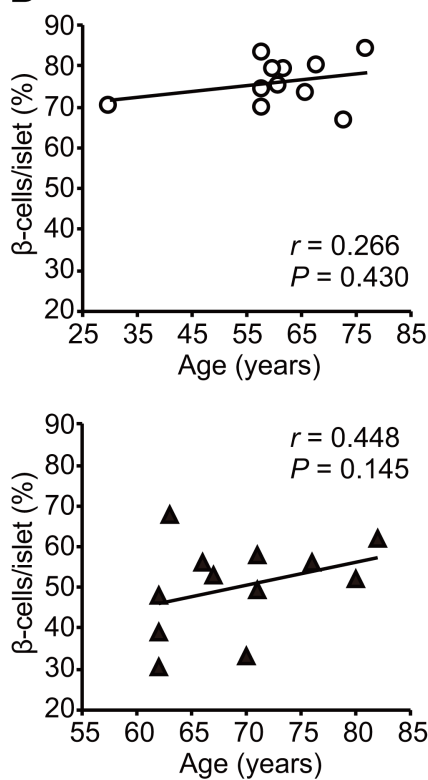
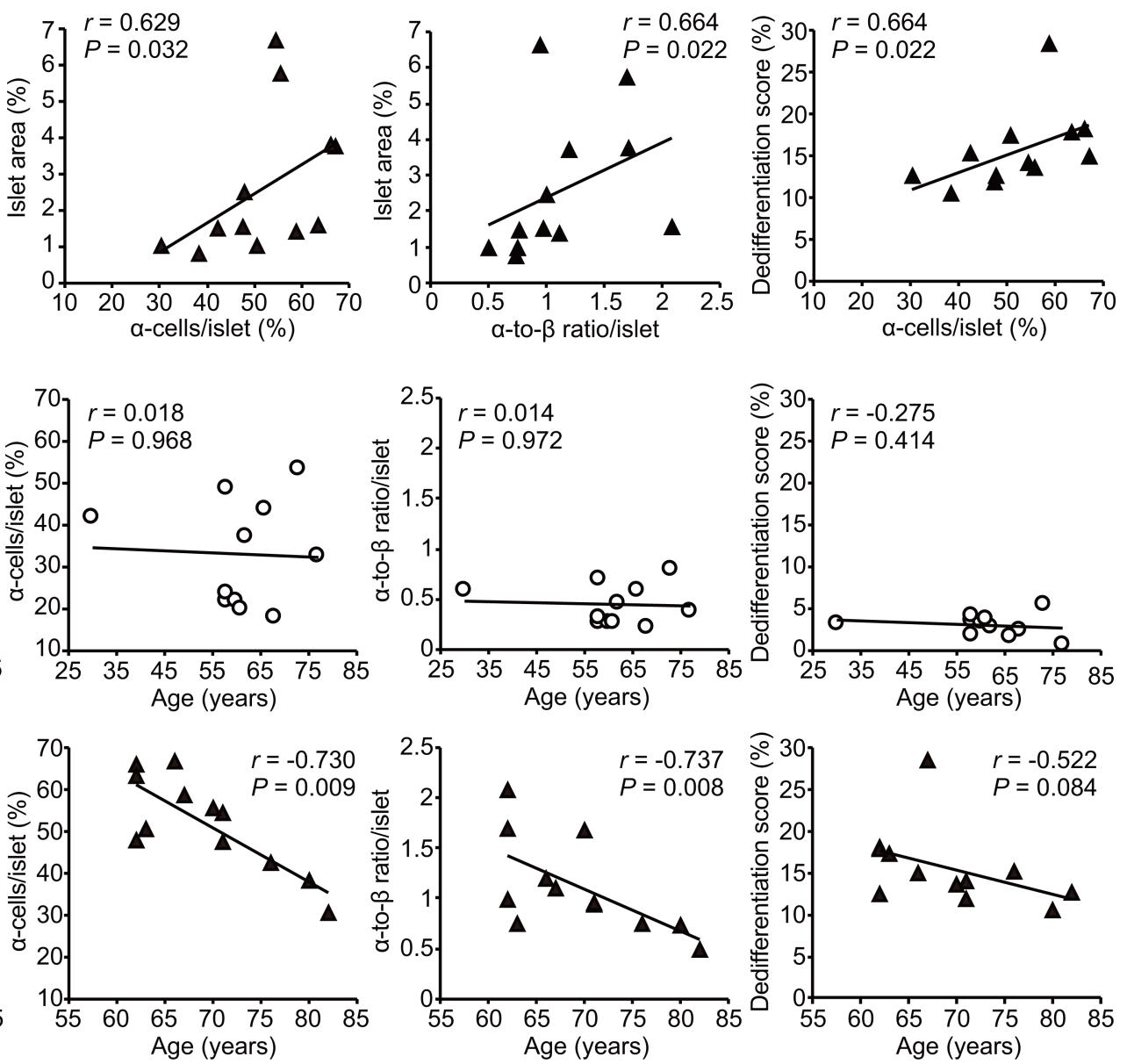

Figure 3. Analysis of correlations of dedifferentiation with islet morphology and age in early-DM subjects. (A) Single regression analysis (Spearman's correlation coefficient) of correlations between islet morphology parameters and islet area with dedifferentiation scores in early-DM subjects ( $n=12$ ). (B) Single regression analysis (Spearman's correlation coefficient) of correlations between age and islet plasticity parameters in non-DM control ( $n=11$ ) and early-DM subjects $(n=12)$. Open circles, non-DM control subjects. Closed triangles, early-DM subjects.

\section{Discussion}

The pathogenesis of $\beta$ cell failure integrates both functional and quantitative (cell number) defects. Islet exerts plasticity in diverse metabolic conditions $(18,19,22)$, and it has been suggested that $\beta$ cells undergo dedifferentiation in type 2 diabetes $(14,16,17,23)$. In light of the human evidence, we sought to elucidate the relevance of dedifferentiation to disease progression during the course of diabetes. Our study using surgically resected pancreatic specimens, taken into consideration with clinical information, demonstrated that islet plasticity in diverse disease conditions, which leads to dedifferentiation, is a pathologic basis of $\beta$ cell failure over the entire course of type 2 diabetes.

From a clinical standpoint, our study had the following potentially novel findings. First, the clinical relevance of dedifferentiation to long-standing progression of diabetes was demonstrated. Second, islet cells undergo dedifferentiation in the early stage of diabetes, in association with $\beta$ cell dysfunction. Finally, a subset of dedifferentiated cells exhibited an exocrine-like phenotype, in association with profound dysfunction. Despite the large deviations of percentage areas of $\beta$ cell, $\alpha$ cell, and islet relative to whole pancreas area, loss of $\beta$ cells and expansion of $\alpha$ cells in individual islets and their correlation with dedifferentiation are the common features of islets of patients with diabetes. Importantly, a link between dedifferentiation and dysfunction was demonstrated in association with disease progression. Despite loss of $\beta$ cells, numbers of islet endocrine cells, as assessed based on ChgA immunoreactivity, were preserved even in subjects with advanced DM, implying an increase in $\beta$ cells that had lost mature identity and the capacity for insulin production. This was supported by transcription factor analysis demonstrating progressive loss of nuclear MAFA and NKX6.1 as insulin-positive cells decreased. In contrast to previous studies $(14,16)$, cells with 
ChgA/Amylase
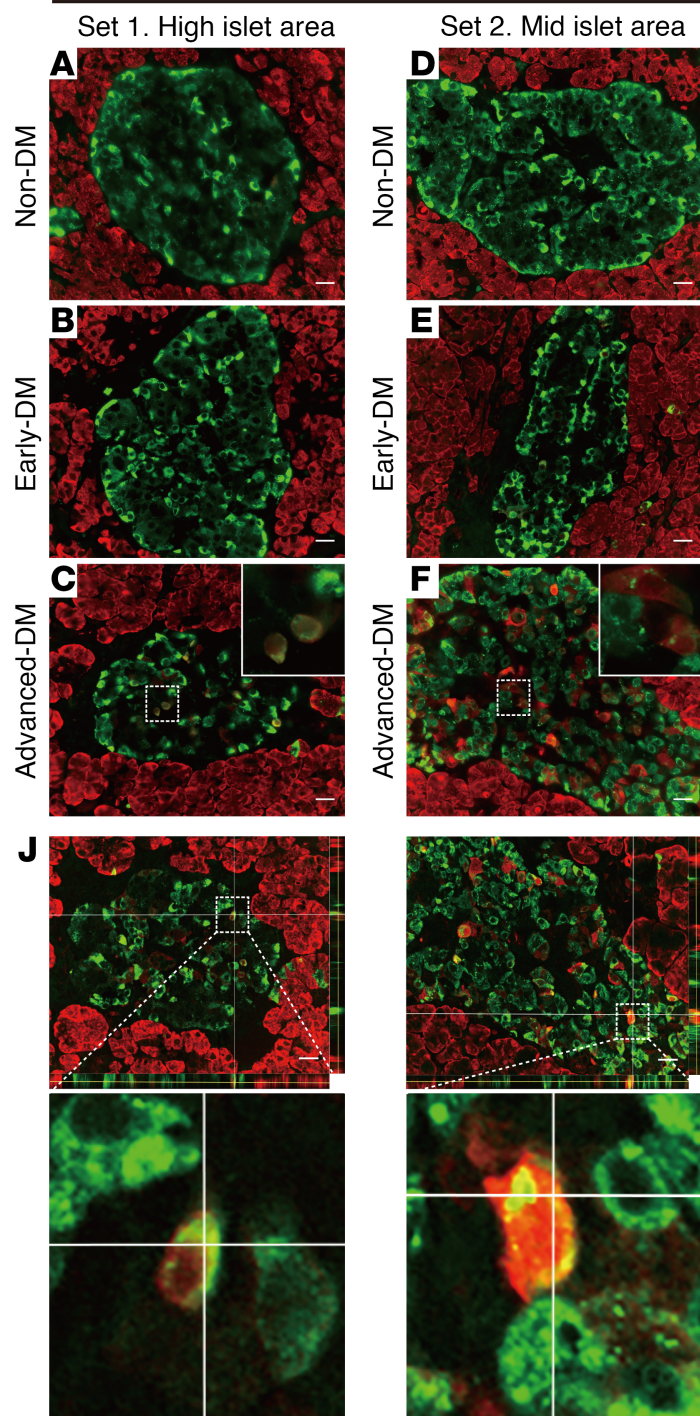
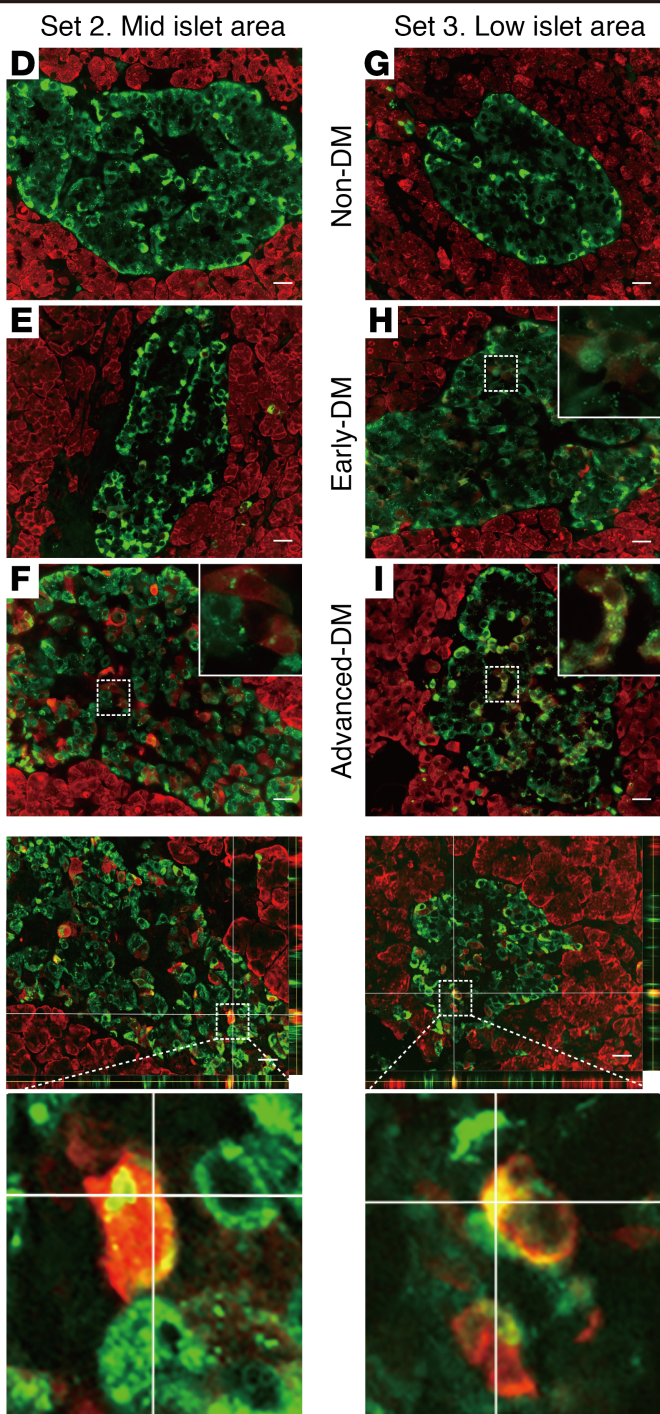
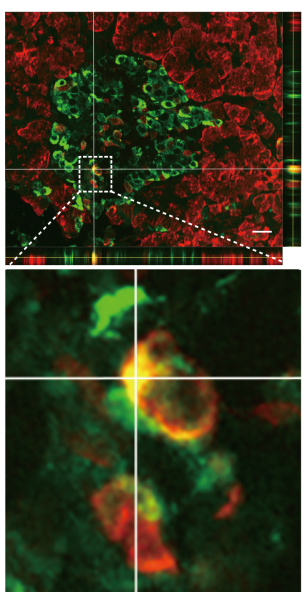

Figure 4. Immunohistochemical evidence of conversion from endocrine to exocrine cell phenotype in failing islets. Islet area-matched male subjects without pancreatic cancer were selected from each group. The 9 subjects were classified into 3 comparison sets according to the fraction of islet area as shown in Supplemental Table 3. Their pancreatic sections were examined. Representative images of pancreatic islets immunostained with ChgA (green) and amylase (red) of 9 subjects in 3 comparison sets representing different islet areas are shown (A-C, D-F, and $\mathbf{G}-\mathbf{I})$. Insets demonstrate representative cells showing immunoreactivity for ChgA and amylase. Scale bar: $20 \mu \mathrm{m}$. (J) Z-stack of pancreatic islets immunostained with ChgA (green) and amylase (red) of advanced-DM. Multiple Z-plane fluorescent images of pancreatic sections of the subjects in $\mathbf{C}, \mathbf{F}$, and I were captured. The representative $Z$-stack images are shown in the same order as in $\mathbf{C}, \mathbf{F}$, and $\mathbf{I}$. The representative islet cells expressing both amylase (red) and ChgA (green) are shown in the lower images with a high magnification. Scale bar: $20 \mu \mathrm{m}$.

mixed $\alpha$ and $\beta$ features, which were partly represented by insulin/Gcg double positivity, were undetectable in our study. This may suggest that $\beta$ cells are unlikely be a source of $\alpha$ cells, at least in Japanese subjects with diabetes. In fact, a recent study reported a similar observation in surgically resected pancreas from Japanese subjects with long-standing diabetes (24).

In rodents, dedifferentiated $\beta$ cells revert to a progenitor-like stage characterized by transcription factor expression $(12,13,25)$. As previously described (14), we were also unable to detect neurogenin 3 immunoreactivity in islets. Aldehyde dehydrogenase 1 isoform A3 (ALDH1A3) has been identified as an alternative progenitor marker in failing islets $(14,26)$. However, given the nature of ALDH1 in cancer progenitor cells (27), we did not assess the significance of ALDH1A3 to avoid implications that would lack the certainty necessary for this analysis. In fact, pancreatic cancer tissues from our cases showed strong immunoreactivities 
for ALDH1A3 (data not shown). Thereby, understanding to what extent islet cells become dedifferentiated was limited. Moreover, we observed that a subset of islet endocrine cells exhibited an exocrine-like phenotype, in association with disease progression. This finding may provide pathologic insight into the irreversible and profound dysfunction in advanced disease. Further work exploring the association between the stage of dedifferentiation and functionality is needed to gain key mechanistic insight into failing islets.

When is dedifferentiation initiated? We observed that $\beta$ cells undergo dedifferentiation, starting early in the disease course. In this regard, a correlation between dedifferentiation and increased $\alpha$ cells was observed in early-DM subjects, and the increasing $\alpha$ cell/islet ratio paralleled expansion of islet area (Figure 3A). As reported previously, nondiabetic subjects with insulin resistance showed increased numbers of $\alpha$ cells and $\beta$ cells with a consequent increase in islet volume $(22,28)$, suggesting that expansion of $\alpha$ cells in early DM could, at least partly, result from preexisting insulin resistance. Therefore, we speculate that when faced with sustained hyperglycemia, islet cells exert plasticity favoring dedifferentiation, possibly potentiated by insulin resistance. In addition, whereas our subject number was small, we did not observe that aging promoted either dedifferentiation or expansion of $\alpha$ cells. This allows us to hypothesize that islet cell plasticity declines with aging, although investigations with a larger number of subjects will be needed to examine this hypothesis.

Loss of maturity through dedifferentiation provides a pathophysiologic link between dysfunction and reduced $\beta$ cell numbers. Despite the evidence that reversal of hyperglycemia can partially restore $\beta$ cell function even in advanced disease (29), the potential for functional recovery declines with disease progression, ultimately leading to irreversible insulin insufficiency (18). Our observation that dedifferentiation scores did not differ between treatments with insulin and sulfonylurea in advanced disease suggest that even decreasing the $\beta$ cell overload by insulin therapy would not reverse dedifferentiation, once diabetes has reached an advanced disease stage. Therefore, intervention in the early disease stage against insulin resistance as well as hyperglycemia reduces metabolic overloading of $\beta$ cells and thereby offers an opportunity for recovery, thus preventing a devastating and irreversible loss of $\beta$ cells $(18,30-32)$. Nonetheless, for $\beta$ cells themselves, adopting dedifferentiation is advantageous. This seemingly "selfish" behavior facilitates the survival of $\beta$ cells that are stressed continuously by metabolic overloads during the long-term course of diabetes.

Our study has limitations. Subjects with various pancreaticobiliary tumors were selected by applying stringent exclusion criteria to reduce potential tumor-related effects on disease conditions and/or progression, and we carefully sectioned the tissues with avoidance of nearby tumors. However, pancreatic cancer in particular may have affected islet plasticity and morphology through indirect mechanisms, which would be a limitation of this study (33).

In conclusion, our observations highlight islet cell dedifferentiation as a mechanism underlying diabetes progression. This makes dedifferentiation a potential target for treatments. Future studies exploring mechanistic insight into islet plasticity with dedifferentiation are warranted and may open new avenues to the prevention and/or reversal of $\beta$ cell failure in type 2 diabetes.

\section{Methods}

Research design. Archived pancreatic samples from 26 Japanese subjects with type 2 diabetes (23 males and 3 females) and 11 nondiabetic control subjects ( 9 males and 2 females), who had undergone partial pancreatectomy (19 PD, 18 DP) were examined for resection of pancreaticobiliary tumors. A total of 29 patients had been operated on at the Department of Gastroenterological Surgery, Yamaguchi University Hospital, Ube, Japan, 8 at the Department of Hepatobiliary and Pancreatic Surgery, Tokyo Medical and Dental University Hospital, Tokyo, Japan. We retrospectively selected subjects according to their medical histories and records.

Description of subjects. All subjects had been overweight (BMI $\geq 25 \mathrm{~kg} / \mathrm{m}^{2}$ ) during adulthood, and none had a family history of diabetes. Patients administered anticancer drugs and glucocorticoids prior to pancreatic resection, those with functional pancreatic endocrine tumors, those with a medical history of pancreatitis and excessive alcohol consumption, and those with renal failure (estimated glomerular filtration rate $<30 \mathrm{~mL} / \mathrm{min} / 1.73 \mathrm{~m}^{2}$ ) were all excluded from this study. Based on fasting and casual blood glucose and $\mathrm{HbA1c}$ measured 1-14 days before pancreatic resection, glycemic status was confirmed to meet the criteria for diabetes or normal glucose tolerance defined by the Japan Diabetes Society (34). One nondiabetic patient whose glycemic status was validated by a $75 \mathrm{~g}$ oral glucose tolerance test lacked an HbA1c measurement. To preclude tumor-related diabetes, only patients who had been diagnosed with type 2 diabetes by 
a diabetologist and had been under medical management for at least 3 years were enrolled. Furthermore, patients with a relative increase in $\mathrm{HbA} 1 \mathrm{c}$ of more than $1 \%(11 \mathrm{mmol} / \mathrm{mol})$ in the $6 \mathrm{months}$ prior to surgery and those with a preoperative $\mathrm{HbA} 1 \mathrm{c}$ of more than $8.6 \%(70 \mathrm{mmol} / \mathrm{mol})$ were excluded. Patients in whom either insulin or insulin secretagogues had been newly administered owing to worsening glycemic control in the 12 months before pancreatic resection were also excluded. Subjects with diabetes were subclassified into 3 disease progression categories, early-DM, advanced-DM, and intermediate-DM, according to medical records. Early-DM was defined as a disease duration of less than 5 years, no medical history of microvascular complications, and never having been treated with insulin and/or sulfonylurea. Advanced-DM was defined as a disease duration of more than 10 years, any medical history of microvascular complications, and continuous treatment with sulfonylurea or insulin for at least 1 year. Intermediate-DM was defined by exclusion from the other categories. In a subset of subjects (5 non-DM, 4 early-DM, 9 advanced-DM, and 3 intermediate-DM), preoperative serum fasting CPR and blood glucose were concomitantly measured. To assess insulin secretory reserve, we calculated the CPI using the following formula: fasting CPR/fasting plasma glucose (35). Features of individual subjects are shown in Supplemental Table 1.

Pancreatic tissue processing and immunohistochemistry. Pancreatic samples obtained from the edge of the surgically resected portion had been fixed, paraffin-embedded, and archived. The tissues were serially sectioned at a thickness of $4 \mu \mathrm{m}$ at 2 random levels and processed for histological analysis. All hematoxylin and eosin-stained pancreatic sections were confirmed by in-house pathologists to contain no pancreatic tissues with tumor elements, pancreatitis and/or autolysis, and immunohistochemistry was then performed. Nuclear counterstaining of sections was performed with DAPI (Vector Laboratories). Histochemical reactions were assessed at the same time in all groups studied, using the same lot of antibodies at dilutions and light exposure times predetermined to maximize sensitivity and minimize nonspecific staining. To identify islet dedifferentiated cells, the tissues were stained with a cocktail of 4 major pancreatic hormones $(4 \mathrm{H}$ : insulin, glucagon, somatostatin, and pancreatic polypeptide) and ChgA according to a previous report (14). All antibodies used for immunohistochemical analysis are listed in Supplemental Table 4. Fluorescence images were captured using a BZ-X710 microscope with BZ-X software (Keyence).

Morphometric analysis. To perform quantitative analyses, we analyzed 2 random sections per subject. We also randomly selected 20 islets with a long diameter of $150-400 \mu \mathrm{m}$ over pancreas area per section and captured them to allow analysis of individual islets. For determination of $\beta$ cell, $\alpha$ cell, and islet areas, the entire pancreatic section was imaged, and the total area of the pancreas was measured with removal of interlobular connective tissue, large blood vessels, and adipocytes. The immunoreactive areas for insulin, glucagon, and ChgA were then determined. $\beta$ cell, $\alpha$ cell, and islet areas were expressed as a percentage of the total pancreatic area, and the ratio of $\alpha$ cell area to $\beta$ cell area was represented as the $\alpha$ cell/ $\beta$ cell ratio. The numbers of insulin-, Gcg-, and ChgA-positive cells per islet were scored according to the DAPI-stained nucleus. The fractions of insulin- and Gcg-positive cells to ChgA-positive cells were represented as $\beta$ cells/islet and $\alpha$ cells/ islet, respectively. The ratio of $\alpha$ cells/islet to $\beta$ cells/islet was represented as the $\alpha$ cell/ $\beta$ cell ratio per islet. The ChgA-positive and $4 \mathrm{H}$-negative cells were scored as dedifferentiated cells, as previously reported (14). We captured islets containing at least 1 dedifferentiated cell and measured percentage of dedifferentiated cells in ChgA-positive cells per islet, representing it as dedifferentiation score. The number of insulin/Gcg double-positive cells per islet was measured in subjects less than 70 years of age whose $\alpha$ cell/ $\beta$ cell ratio per islet score was within the mean $\pm 0.5 \mathrm{SD}$. In subjects whose dedifferentiation scores were within the mean \pm $0.5 \mathrm{SD}$, we scored insulin-positive cells stained for nuclear MAFA and NKX6.1. For quantitative analysis of insulin/Gcg double-positive cells and transcription factors in $\beta$ cells, at least 2000 insulin-positive cells per subject were examined. Supplemental Table 1 shows the details of the analyzed subjects. All quantitative measurements were conducted using BZ-X software in a blinded fashion by 2 in-house independent investigators.

Statistics. Quantitative data are presented as means \pm SD. Significant differences were evaluated by 1-way ANOVA followed by Bonferroni's post hoc test. To assess correlations among morphometric data and with clinical parameters, single regression analysis was carried out with Spearman's correlation coefficient. A $P$ value of less than 0.05 was considered significant. All statistical analyses were performed with GraphPad Prism software version 7.

Study approval. The collection and use of clinical data, followed by analysis of pancreatic samples, were approved by the ethics review board of Yamaguchi University Hospital (approval number H29-112, H30-176) and Tokyo Medical and Dental University Hospital (approval number M2000-1890). All subjects provided appropriate written informed consent. 


\section{Author contributions}

KAS and $\mathrm{K}$ Tanabe prepared the manuscript. K Tanabe and YT designed this study. KAS, K Tanabe, HM, RH, T Takagi, TF, T Takeuchi, RB, MH, K Takeda, and SO obtained the data. KAS and K Tanabe wrote the manuscript. KAS, K Tanabe, and YT edited the manuscript. YT reviewed the manuscript. HM, AK, ST, MT, and HN performed partial pancreatectomy procedures. KAS, K Tanabe, YH, RH, T Takeuchi, WN, and EI performed pathologic analyses of pancreatic specimens. TY, YO, EI, HN, and YT contributed to interpretation of the data. All authors were involved in discussing the results and providing commentary on the manuscript. K Tanabe and YT had full access to all of the data in the study and take responsibility for data integrity and accuracy of data analysis. YT is the guarantor of this work.

\section{Acknowledgments}

The authors thank all staff members of the Division of Endocrinology, Metabolism, Hematological Sciences and Therapeutics, Yamaguchi University Graduate School of Medicine, and the Department of Diagnostic Pathology, Yamaguchi University Hospital, for their contributions. This study was supported by a Grant-in-Aid for Scientific Research (grant number 16K09752 and 20K08887) (to K Tanabe) and a Grant-in-Aid for Scientific Research (grant number 19H03710) from Takeda Science Foundation (to YT). KAS is supported by a Grant-in-Aid for Scientific Research (grant number 19K07506), a Japan Diabetes Society Junior Scientist Development Grant supported by Novo Nordisk Pharma Ltd., a Grant for Front Runner of Future Diabetes Research, and Grants for young researchers from the Japan Association for Diabetes Education and Care and from MSD Life Science Foundation, Public Interest Incorporated Foundation. The funding agencies had no role in study design, data collection and interpretation, or the decision to submit the work for publication.

Address correspondence to: Katsuya Tanabe, 1-1-1 Minami-kogushi, Ube, Yamaguchi, 755-8505, Japan. Phone: 81.836.2252; Email: ktanabe@yamaguchi-u.ac.jp.

1. Weir GC, Bonner-Weir S. Islet $\beta$ cell mass in diabetes and how it relates to function, birth, and death. Ann NY Acad Sci. 2013;1281:92-105.

2. Nolan CJ, et al. Type 2 diabetes across generations: from pathophysiology to prevention and management. Lancet. 2011;378(9786):169-181.

3. UK Prospective Diabetes Study (UKPDS) Group. Intensive blood-glucose control with sulphonylureas or insulin compared with conventional treatment and risk of complications in patients with type 2 diabetes (UKPDS 33). Lancet. 1998;352(9131):837-853.

4. UK Prospective Diabetes Study (UKPDS) Group. Effect of intensive blood-glucose control with metformin on complications in overweight patients with type 2 diabetes (UKPDS 34). Lancet. 1998;352(9131):854-865.

5. Poitout V, Robertson RP. Glucolipotoxicity: fuel excess and beta-cell dysfunction. Endocr Rev. 2008;29(3):351-366

6. Prentki M, Nolan CJ. Islet beta cell failure in type 2 diabetes. J Clin Invest. 2006;116(7):1802-1812.

7. Butler AE, et al. Beta-cell deficit and increased beta-cell apoptosis in humans with type 2 diabetes. Diabetes. 2003;52(1):102-110.

8. Mizukami H, et al. Involvement of oxidative stress-induced DNA damage, endoplasmic reticulum stress, and autophagy deficits in the decline of $\beta$-cell mass in Japanese type 2 diabetic patients. Diabetes Care. 2014;37(7):1966-1974.

9. Rahier J, et al. Pancreatic beta-cell mass in European subjects with type 2 diabetes. Diabetes Obes Metab. 2008;10(suppl 4):32-42.

10. Kahn SE, et al. The beta cell lesion in type 2 diabetes: there has to be a primary functional abnormality. Diabetologia. 2009;52(6):1003-1012

11. Dai C, et al. Stress-impaired transcription factor expression and insulin secretion in transplanted human islets. J Clin Invest. 2016;126(5):1857-1870.

12. Talchai C, et al. Pancreatic $\beta$ cell dedifferentiation as a mechanism of diabetic $\beta$ cell failure. Cell. 2012;150(6):1223-1234.

13. Wang $Z$, et al. Pancreatic $\beta$ cell dedifferentiation in diabetes and redifferentiation following insulin therapy. Cell Metab. 2014;19(5):872-882

14. Cinti F, et al. Evidence of $\beta$-cell dedifferentiation in human type 2 diabetes. J Clin Endocrinol Metab. 2016;101(3):1044-1054.

15. Marselli L, et al. Are we overestimating the loss of beta cells in type 2 diabetes? Diabetologia. 2014;57(2):362-365.

16. Spijker HS, et al. Loss of $\beta$-cell identity occurs in type 2 diabetes and is associated with islet amyloid deposits. Diabetes. 2015;64(8):2928-2938.

17. Sun J, et al. $\beta$-Cell dedifferentiation in patients with T2D with adequate glucose control and nondiabetic chronic pancreatitis. J Clin Endocrinol Metab. 2019;104(1):83-94.

18. White MG, et al. Type 2 diabetes: the pathologic basis of reversible $\beta$-cell dysfunction. Diabetes Care. 2016;39(11):2080-2088.

19. Remedi MS, Emfinger C. Pancreatic $\beta$-cell identity in diabetes. Diabetes Obes Metab. 2016;18(suppl 1):110-116.

20. White MG, et al. Expression of mesenchymal and $\alpha$-cell phenotypic markers in islet $\beta$-cells in recently diagnosed diabetes. Diabetes Care. 2013;36(11):3818-3820.

21. Mezza T, et al. Insulin resistance alters islet morphology in nondiabetic humans. Diabetes. 2014;63(3):994-1007.

22. Mezza T, et al. $\beta$-cell fate in human insulin resistance and type 2 diabetes: a perspective on islet plasticity. Diabetes. 2019;68(6):1121-1129. 
23. Guo S, et al. Inactivation of specific $\beta$ cell transcription factors in type 2 diabetes. J Clin Invest. 2013;123(8):3305-3316

24. Yoneda $S$, et al. Predominance of $\beta$-cell neogenesis rather than replication in humans with an impaired glucose tolerance and newly diagnosed diabetes. J Clin Endocrinol Metab. 2013;98(5):2053-2061.

25. Taylor BL, et al. Nkx6.1 is essential for maintaining the functional state of pancreatic beta cells. Cell Rep. 2013;4(6):1262-1275.

26. Kim-Muller JY, et al. Aldehyde dehydrogenase 1a3 defines a subset of failing pancreatic $\beta$ cells in diabetic mice. Nat Commun . 2016;7:12631

27. Ginestier C, et al. ALDH1 is a marker of normal and malignant human mammary stem cells and a predictor of poor clinical outcome. Cell Stem Cell. 2007;1(5):555-567.

28. Inaishi J, et al. Effects of obesity and diabetes on $\alpha$ - and $\beta$-cell mass in surgically resected human pancreas. J Clin Endocrinol Metab. 2016;101(7):2874-2882.

29. Savage PJ, et al. Diet-induced improvement of abnormalities in insulin and glucagon secretion and in insulin receptor binding in diabetes mellitus. J Clin Endocrinol Metab. 1979;48(6):999-1007.

30. Ishida E, et al. Pair feeding, but not insulin, phloridzin, or rosiglitazone treatment, curtails markers of $\beta$-cell dedifferentiation in db/db mice. Diabetes. 2017;66(8):2092-2101.

31. Ferrannini E. The stunned beta cell: a brief history. Cell Metab. 2010;11(5):349-352.

32. Weng J, et al. Effect of intensive insulin therapy on beta-cell function and glycaemic control in patients with newly diagnosed type 2 diabetes: a multicentre randomised parallel-group trial. Lancet. 2008;371(9626):1753-1760.

33. Wang Y, et al. Paraneoplastic $\beta$ cell dedifferentiation in nondiabetic patients with pancreatic cancer. J Clin Endocrinol Metab. 2020;105(4):dgz224.

34. Committee of the Japan Diabetes Society on the Diagnostic Criteria of Diabetes Mellitus, et al. Report of the committee on the classification and diagnostic criteria of diabetes mellitus. J Diabetes Investig. 2010;1(5):212-228

35. Yamada Y, et al. SUIT, secretory units of islets in transplantation: an index for therapeutic management of islet transplanted patients and its application to type 2 diabetes. Diabetes Res Clin Pract. 2006;74(3):222-226. 